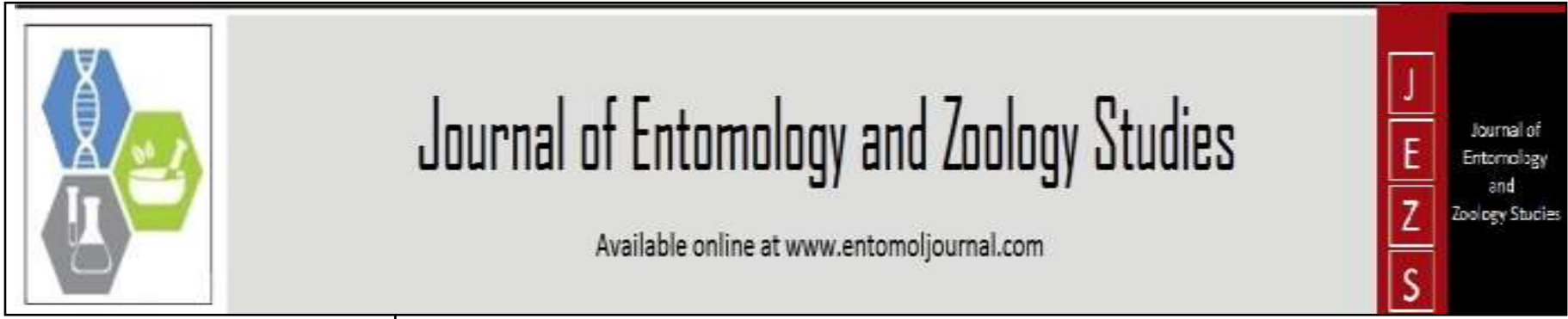

E-ISSN: 2320-7078

P-ISSN: 2349-6800

www.entomoljournal.com

JEZS 2021; 9(1): 1977-1981

(C) $2021 \mathrm{JEZS}$

Received: 03-11-2020

Accepted: 16-12-2020

\section{Sandhya Bhavani}

Assistant Professor, Department

of Clinics, Madras Veterinary

College, Chennai, Tamil Nadu,

India

\section{S Kavitha}

Professor and Head, Department

of Veterinary Clinical Medicine,

Madras Veterinary College,

Chennai, Tamil Nadu, India

\section{Sumathi}

Assistant Professor, Department of Veterinary Clinical Medicine,

Madras Veterinary College,

Chennai, Tamil Nadu, India

\section{Abid Ali Bhat}

Department of Veterinary Clinical Medicine, College of Veterinary Medicine and Animal Sciences, University of Gondar, Ethiopia

\section{Abdominal ultrasonography in canine inflammatory bowel disease: A short study}

\author{
M Sandhya Bhavani, S Kavitha, D Sumathi and Abid Ali Bhat
}

DOI: $\underline{\text { https://doi.org/10.22271/j.ento.2021.v9.i1ab.8421 }}$

Abstract

Ultrasonography is a noninvasive and inexpensive tool to examine the gastrointestinal tract of small animals with chronic gastrointestinal signs. It plays a major role in the recognition, diagnosis and monitoring of inflammatory bowel disease (IBD) in human patients. The present study was undertaken to record the ultrasonographic changes associated with canine IBD. Thirty three dogs confirmed with idiopathic IBD were included in the study and subjected to detailed abdominal ultrasonography. This study revealed that dogs with IBD variably have a normal to mildly thickened intestinal wall thickness. Secondary changes recorded in the IBD dogs were gas filled intestinal loops, hypermotility of intestinal loops, dilation of the lumen and mesenteric lymphnode enlargement. In conclusion, intestinal wall measurements do not appear to establish a diagnosis of intestinal inflammation in canine IBD in contrast to humans where ultrasonography has a significant role in establishing the diagnosis.

Keywords: Canine, IBD, Intestinal wall thickness, ultrasonography, secondary changes

\section{Introduction}

Ultrasonography (USG) is one of the important imaging modalities used in the diagnosis of gastrointestinal diseases in small animal practice. Moreover, being noninvasive and safer, USG is routinely used in animal practice to examine the gastrointestinal tract (GIT) in acute as well as chronic gastrointestinal diseases. Abdominal radiography is an important part of baseline examination for patients with vomiting and diarrhea. However, ultrasonographic examination is comparatively superior for detecting infiltrative intestinal diseases. USG is also helpful in differentiating inflammatory from neoplastic diseases of gastrointestinal tract, and thereby helpful in the selection of appropriate treatment protocol. The USG examination of gastrointestinal tract (GIT) reveals details such as focal or segmental changes, relative thickness of the layers, total wall thickness, abnormal hypo or hyperechogenicity etc. which characteristically change in the presence of disease. Among these changes intestinal wall thickness has been an important criterion for determining clinical disease activity and thus bowel wall thickness is useful as a diagnostic marker and monitoring tool ${ }^{[1]}$. Other frequent sonographic findings are fibrofatty proliferation of the mesentery enlarged lymph nodes, and complications such as narrowing of the lumen with prestenotic dilatation, abscesses, and fistulas. Since very little work on canine IBD has been carried out in India and the data on ultrasonographic changes of intestinal inflammation are lacking, the present study was conducted to record the ultrasonograhic changes associated with canine IBD.

\section{Materials and Method \\ IBD dogs and control}

The clinical cases suffering from chronic vomiting, chronic diarrhoea, weight loss, tenesmus, melena and hematochezia were included in the study. Sixty seven dogs included in the study were subjected to basic diagnostic evaluation. Out of $67 \mathrm{dogs}$, Thirty four (34) dogs were excluded from the study due to exocrine pancreatic insufficiency (6), owners refusal to perform endoscopy (12), infestation of Trichuris vulpis which was evident only after the colonoscopic procedure as the faecal examination was negative (4), rectal polyp (2), intestinal lymphoma (1), foreign body (1) and positive for Helicobacter spp. from intestinal biopsy samples by PCR (8). Cases were confirmed as IBD based on histopathologic examination (endoscopic mucosal biopsy specimen) as per World Small Animal Veterinary Association histopathologic guidelines. Thirty three (33) dogs confirmed as cases of IBD were subjected to USG examination.
Corresponding Author: M Sandhya Bhavani

Assistant Professor, Department of Clinics, Madras Veterinary College, Chennai, Tamil Nadu, India 
The control group consisted of ten apparently (10) healthy dogs brought for routine health checkups and vaccination. Written consent was taken from the Owners during the study period.

\section{Ultrasonography procedure}

All the confirmed IBD dogs were subjected to complete ultrasonographic examination of abdomen to assess the gastrointestinal tract with ultrasound scanner (MyLab60, Esaote, Genova, Italy) using high frequency probe (7.5$10 \mathrm{MHz}$ ) as per standard procedure ${ }^{[2]}$.

The GI tract was evaluated for wall thickness, appearance of wall layers, luminal contents and diameter as per previous study ${ }^{[3]}$. Wall thickness was measured from the inner luminal interface to the outer serosal surface. It was considered normal if within published reference ranges (stomach: 2-5 $\mathrm{mm}$, duodenum: 3-6 $\mathrm{mm}$ depending on body weight, jejunum: 2-5 mm depending on body weight, ileum: $2-4 \mathrm{~mm}$, and colon: $2-3 \mathrm{~mm}$ ). Wall layers were considered normal if all layers were visible and were of normal echogenicity. Luminal diameter of the stomach and small intestine was assessed subjectively. If bowel dilatation was present, the affected segment was followed to identify an obstructing lesion. If gastric distension was noted, the pyloric area was carefully evaluated for obstruction. Mesenteric lymph nodes, if visible, were measured and assessed for shape and echogenicity. Lymph node with the thickness was less than 7$8 \mathrm{~mm}$ was considered normal.

\section{Statistical analysis}

Data were presented as the mean \pm standard error (SE). Comparison of the mean of similar parameters was performed by two independent samples t-test. Statistical analysis was performed using SPSS 23.0 and the level of significance was considered $P<0.05$.

\section{Results and Discussion}

Idiopathic inflammatory bowel disease (IBD) is a common cause of chronic diarrhoea in dogs described as the infiltration of inflammatory cells in the intestinal walls ${ }^{[4]}$. The diagnosis of IBD is confirmed after exclusion of diseases causing gastrointestinal signs (parasitic infection, exocrine pancreatic insufficiency, tumours, foreign bodies etc) ${ }^{[5]}$ and histopathologic examination of endoscopic mucosal biopsy specimens [6]. Ultrasonography is often used as the first diagnostic tool to differentiate inflammatory from neoplastic infiltration which is crucial to choose appropriate treatment strategies ${ }^{[7]}$. However, in humans, gastro intestinal ultrasound (GIUS) provides an objective assessment of inflammation especially those with Crohn's disease ${ }^{[8]}$. Gastrointestinal USG complements conventional endoscopy and crosssectional imaging and can be performed at the point of care to accomplish clinical decision-making and optimise patient management ${ }^{[9]}$.

The most prominent and most important parameter that indicates inflammatory activity in human IBD (Crohns disease-CD and ulcerative colitis) is an increase in bowel wall thickness ${ }^{[9]}$. However, in our study, it was observed that there was no difference in the wall thickness of various segments of gastrointestinal tract between normal and IBD dogs. The Mean \pm S.E values of Ultrasonographic Gastrointestinal wall thickness in dogs with IBD are presented in Table 1. On an individual case analysis, mild thickening was recorded in stomach and duodenum in 3 and 5 cases while in jejunum and colon in 6 cases (Fig. 1, 2, 3). Among the dogs with colon inflammation, $3 \mathrm{dogs}$ had colon wall thickness of 4-6 mm while other 3 dogs had wall thickness of 7- 8mm (Fig.4). Furthermore, in 3 cases, the wall of the entire gastrointestinal tract was thickened including mucosal layer. The findings were similar to previous studies ${ }^{[7,10,11]}$ which recorded only mild inflammation and they observed that there are no significant differences in wall thickness in dogs with IBD compared to normal dogs. It was opined that measurements of intestinal wall thickness have not been found to be specific or sensitive for the diagnosis of idiopathic IBD in dogs ${ }^{[10]}$.

Table 1: Mean \pm S.E values of Ultrasonographic Gastrointestinal wall thickness in dogs with IBD

\begin{tabular}{|c|c|c|c|}
\hline $\begin{array}{c}\text { Parameters } \\
\text { Wall thickness } \\
(\mathrm{mm}) \\
\end{array}$ & $\begin{array}{c}\text { Group I - } \\
\text { Control } \\
(n=10)\end{array}$ & $\begin{array}{c}\text { Group II - } \\
\text { IBD } \\
(\mathbf{n}=\mathbf{3 3}) \\
\end{array}$ & t value \\
\hline Stomach & $3.17 \pm 0.05$ & $3.26 \pm 0.12$ & $-0.397^{\mathrm{NS}}$ \\
\hline Duodenum & $4.19 \pm 0.07$ & $4.27 \pm 0.17$ & $-0.238^{N S}$ \\
\hline Jejunum & $3.27 \pm 0.08$ & $3.33 \pm 0.13$ & $0.264^{\mathrm{NS}}$ \\
\hline Colon & $1.92 \pm 0.14$ & $2.89 \pm 0.26$ & $-1.098^{\mathrm{NS}}$ \\
\hline $\begin{array}{l}\text { *- Statistically sigr } \\
\text { NS- Statisticallyno }\end{array}$ & ificant & $\begin{array}{l}<0.01) \\
<0.05) \\
>0.05\end{array}$ & \\
\hline
\end{tabular}

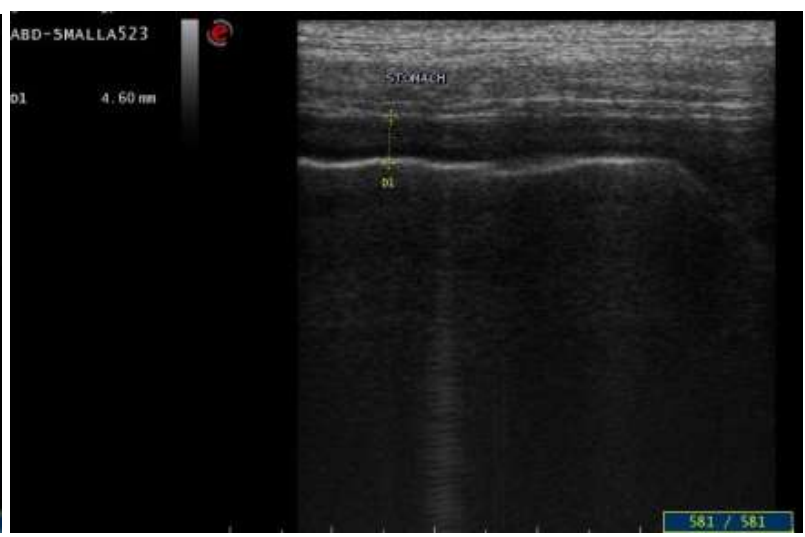

b. Stomach - IBD dog with increased wall thickness

Fig 1: Ultrasonography of stomach 


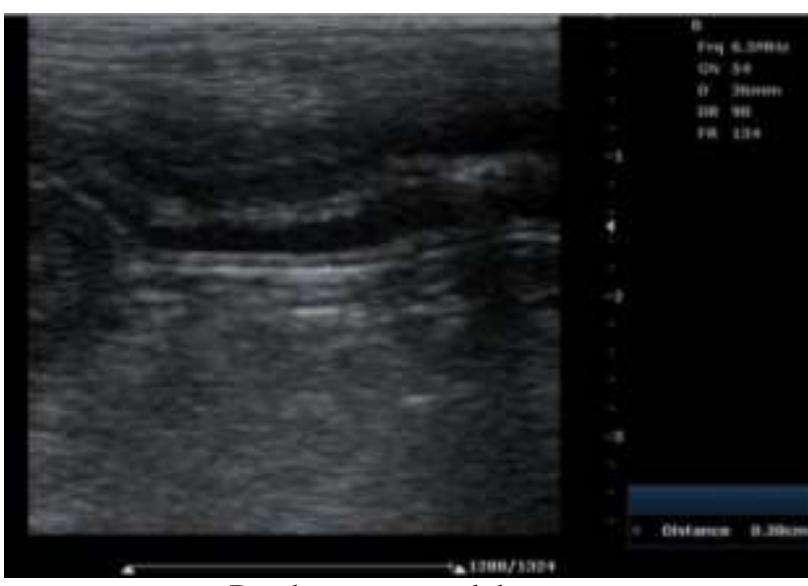

a. Duodenum -normal dog

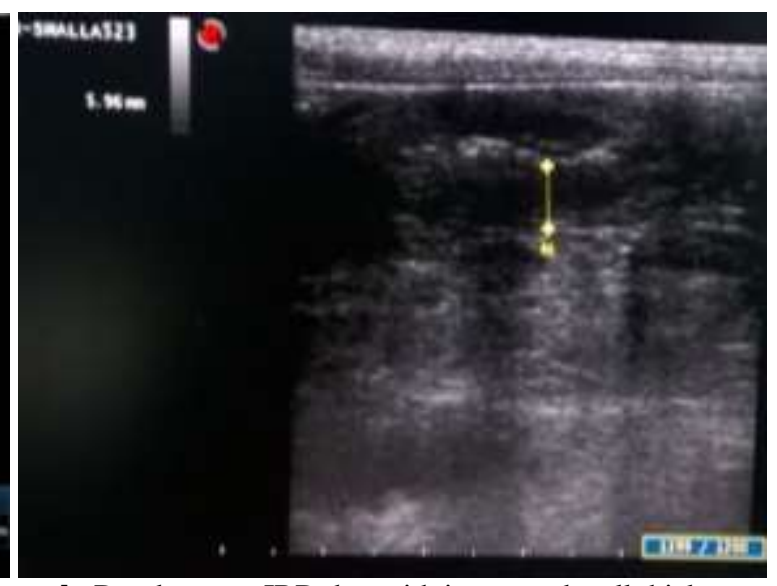

b. Duodenum - IBD dog with imcreased wall thickness

Fig 2: Ultrasonography of duodenum

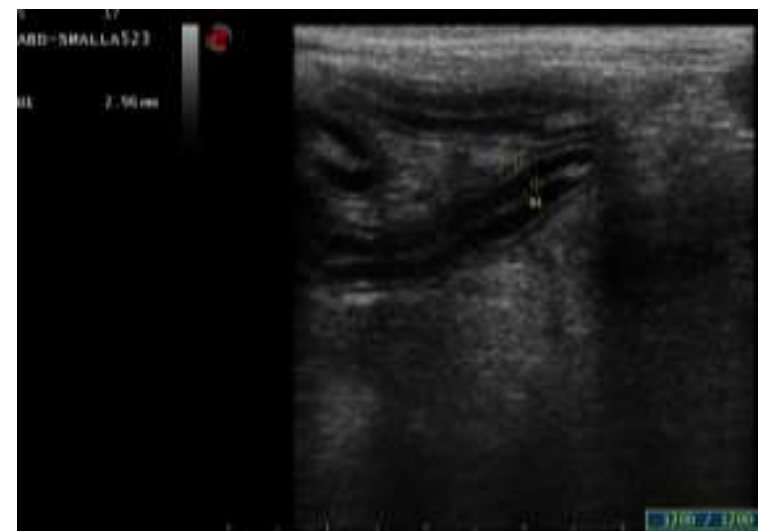

a. Jejunum - IBD dog with normal wall thickness

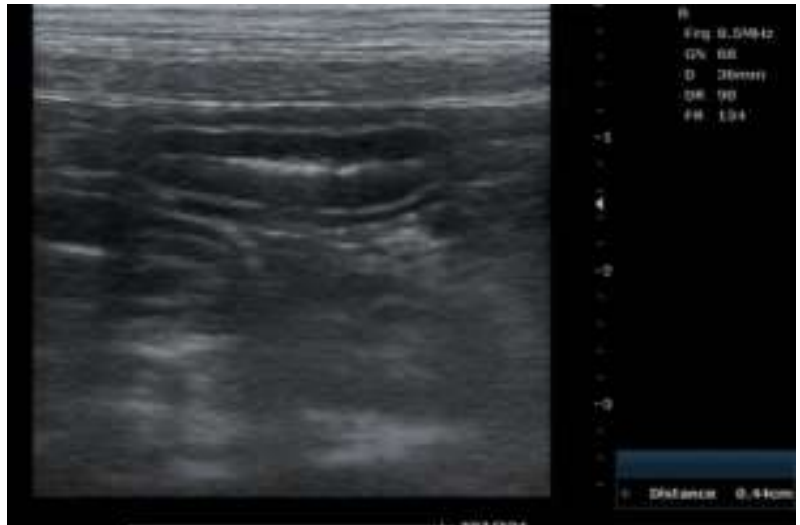

b. Jejunum - IBD dog with increased wall thickness

Fig 3: Ultrasonography of jejunum

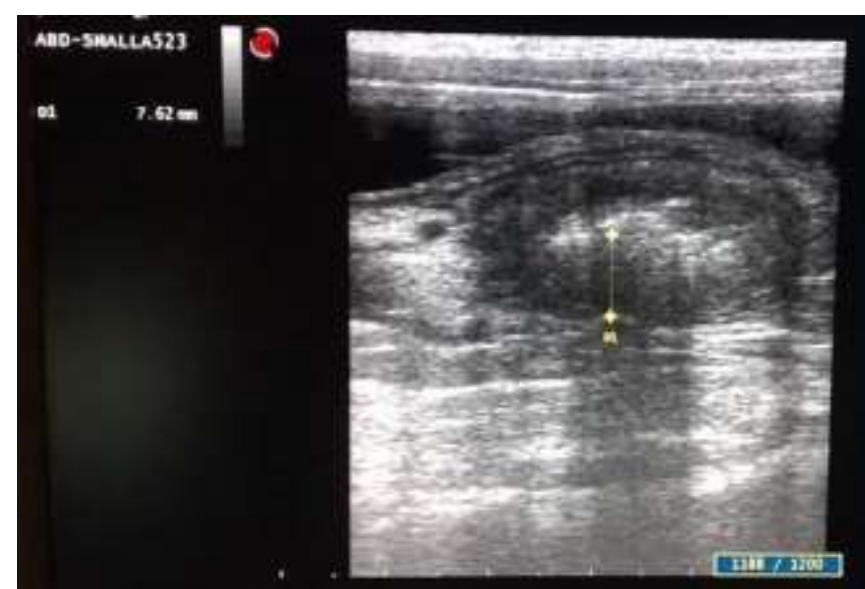

a. Colon- IBD dog with increased wall thickness

Fig 4: Ultrasonography of colon

There are several possible explanations for an apparently normal wall thickness in dogs with IBD. It was suggested that the number of infiltrating cells was not sufficient to cause an enlargement of the wall, although clinical signs are seen ${ }^{[10]}$. Further, villus atrophy accompanying the inflammation may have reduced wall thickness. In one study it was opined that that the inflammatory bowel disease of canine and humans does not resemble closely ${ }^{[11]}$. This may be due to variation in the pathogenesis of bowel wall thickening between the two species. Dogs have more subtle lesions when compared to humans where in granulomatous or fistulating disease is observed. It was also suggested that in humans the presence of submucosal edema may have caused extremely thickened bowel wall with active disease ${ }^{[13]}$.

In this study, normal intestinal wall layering and mucosal echogenicity were recorded in majority cases. Loss of wall layering was noticed in only 4 cases. These findings were consistent with previous studies $\left.{ }^{14} 15\right]$ which showed that bowel wall layering can appear normal in the face of inflammation but may also have a mild loss of definition.

The most common secondary changes associated with IBD observed in the present study were gas filled intestinal loops and hypermotility of intestinal loops (15 cases each), followed by Dilation of lumen with luminal contents in (10 cases) (Fig.5). Mesenteric lymph nodes were found to be enlarged in 16 cases. The size of lymph nodes ranged from $10 \mathrm{~mm}$ to 15 $\mathrm{mm}$. Free fluid in the peritoneum was recorded in 4 cases. Very few data are available on the secondary changes associated with canine IBD. Those previous studies suggested that secondary findings of free fluid, pancreas edema and intestinal dilation were labile and more important in the assessment of therapeutic success ${ }^{[11,16]}$. One study reported that the presence of secondary abnormalities of the intestines such as free abdominal fluid, edema of the pancreas and distended bowel segments are observed in dogs with proteinlosing enteropathy, although it is uncommon in dogs with inflammatory bowel disease ${ }^{[15]}$. 


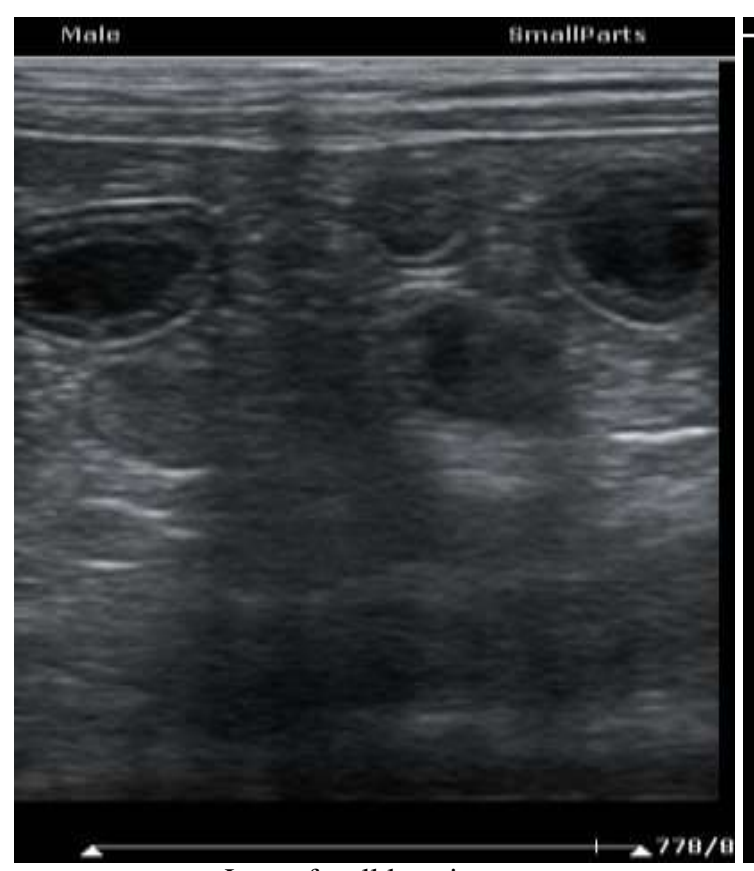

a. Loss of wall layering

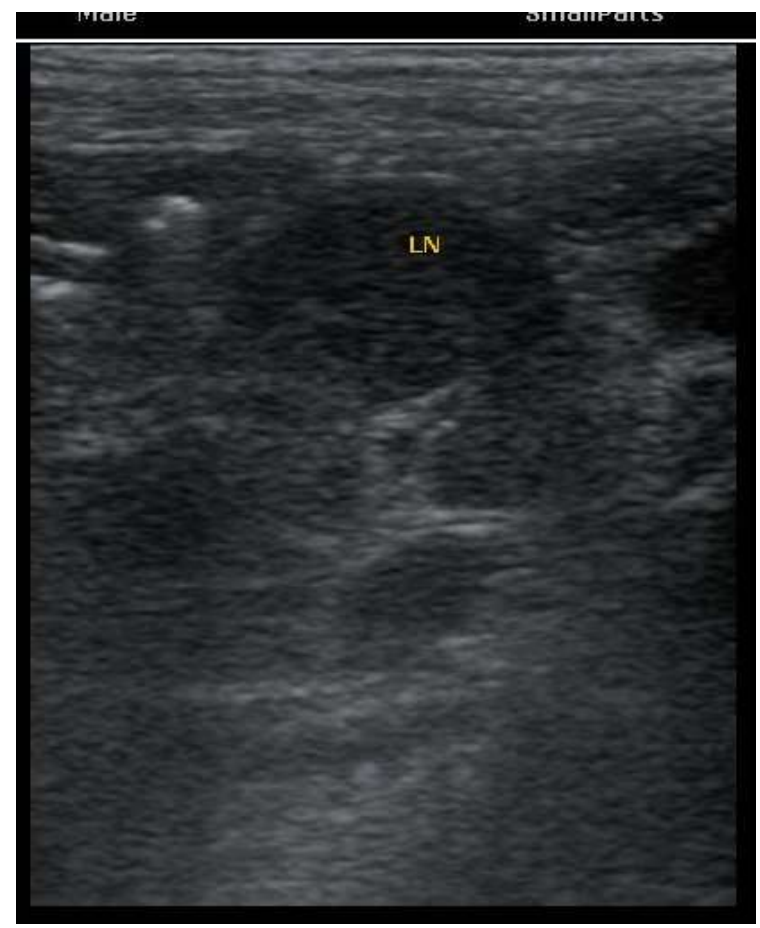

c. Enlarged mesenteric lymphnode

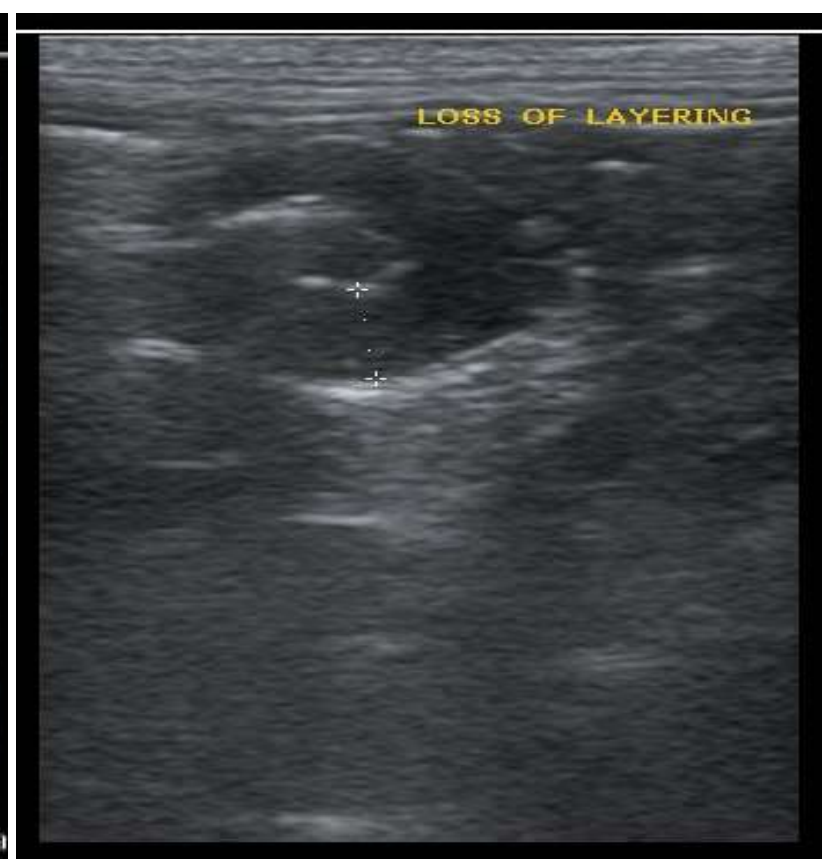

b. Distended loops with contents

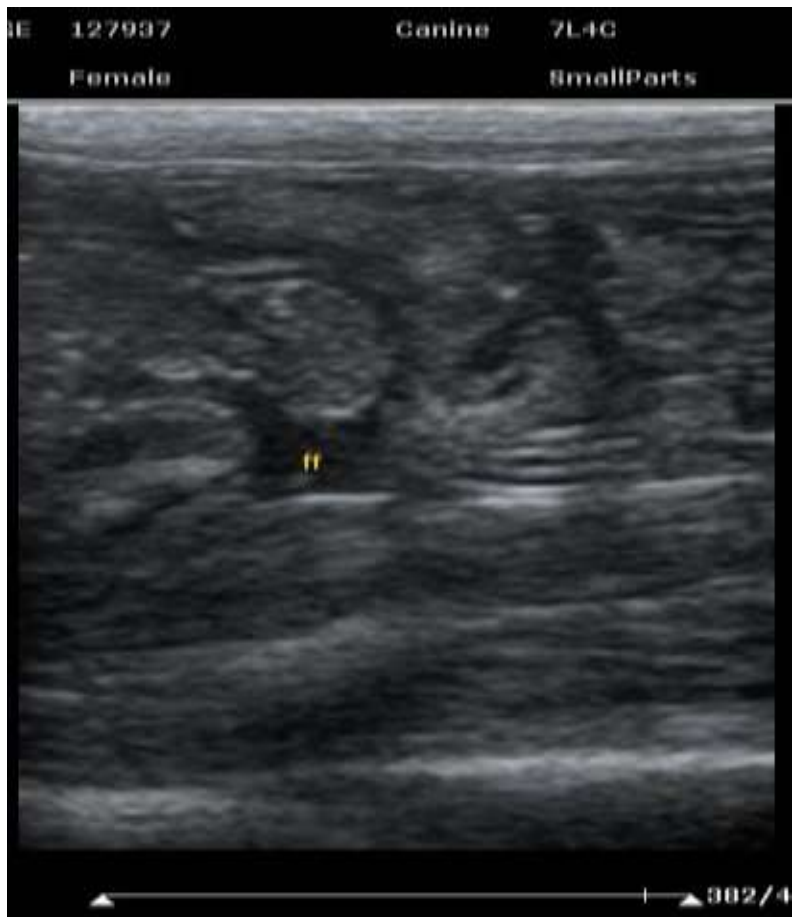

d. Free fluid in the peritoneum

Fig 5: Ultrasonography - secondary changes due to IBD

In summary, several studies have shown that the most important intestinal ultrasonographic parameters to determine disease activity are bowel wall thickness. It is important to note that sonographically normal-appearing intestinal wall does not rule out the presence of inflammation. Moreover, it is to be noted that dogs with IBD may have a normal to mildly thickened small intestinal wall thickness along with few secondary changes. The findings are consistent with previously reported studies on canine IBD. Since the study was conducted with a small number of cases, a larger study is needed to investigate the role of ultrasound in Canine IBD as in human patients.

\section{Conclusion}

Gastrointestinal ultrasound is highly useful in human patients suffering from Crohn's disease (human IBD). However, this study revealed non-significant changes in dogs with IBD. Therefore ultrasound may be of limited use in the diagnosis of canine IBD.

\section{Acknowledgement}

Authors are thankful to the Department of Veterinary Clinical Medicine faculties for their support and help throughout the research.

\section{References}

1. Haber HP, Busch A, Ziebach R. Ultrasonographic findings corresponding to clinical, endoscopic, and histologic findings in inflammatory bowel disease and other enterocolitides. Journal of Ultrasound in Medicine, 2002;21:375-382.

2. Citi S, Chimenti T, Marchetti V, Millanta F, Mannucci T. 
Micronodular Ultrasound Lesions In The Colonic Submucosa Of 42 Dogs And 14 Cats. Veterinary Radiology and Ultrasound.2013;54(6):646-651.

3. Leib MS, Larson MM, Grant DC, Monroe WE, Troy GC. Diagnostic Utility of abdominal ultrasonography in dogs with chronic diarrhoea. Journal of Veterinary Internal Medicine 2012;26:1288-1294.

4. Ristic ME, Stidworthy MF. Two cases of severe irondeficiency anaemia due to inflammatory bowel disease in the dog. Journal of Small Animal Practice 2002;43:80-83.

5. Zentek J, Hall EJ, German A, Haverson K, Bailey M, Rolfe $\mathrm{V}$ et al. Morphology and immunopathology of the small and large intestine in dogs with non-specific dietary sensitivity. Journal of Nutrition 2002;132:1652S-1654S

6. Washabau R, Day M, Willard M, Hall EJ, Jergens AE, Mansell $\mathrm{J}$ et al. ACVIM Consensus statement: Endoscopic, biopsy and histopathologic guidelines for the evaluation of gastrointestinal inflammation in companion animals. Journal of Veterinary Internal Medicine 2010;24:10-26.

7. Penninck DG. Gastrointestinal tract. In: Penninck D.G. and M.A. d'Anjou (eds). Atlas of small animal ultrasonography. Blackwell Publishers, Ames 2008, 281319.

8. Calabrese E, Maaser C, Zorzi F. Bowel Ultrasonography in the Management of Crohn's Disease. A Review with Recommendations of an International Panel of Experts. Inflammatory Bowel Disease 2016;22:1168-83.

9. Bryant RV, Friedman AB, Wright EK, Taylor KM, Begun J, Maconi G et al. Gastrointestinal ultrasound in inflammatory bowel disease: an underused resource with potential paradigm-changing application. Gut, 2018;67(5):973-985. doi:10.1136/gutjnl-2017-315655

10. Rudorf H, van Schaik GO,'Brien RT, Brown PJ, Barr FJ, Hall EF. Ultrasonographic evaluation of the thickness of the small intestinal wall in dogs with inflammatory bowel disease. Journal of Small Animal Practice 2005;46:322326.

11. Gaschen L, Kircher P, Allenspach K. Comparison of ultrasonographic findings with clinical activity index (CIBDAI) and diagnosis in dogs with chronic enteropathies. Veterinary Radiology and Ultrasound, 2008;49(1):56-64.

12. Suchodolski JS, Steiner JM, Evans R. Comparison of Oral Prednisone and Prednisone Combined with Metronidazole for Induction Therapy of Canine Inflammatory Bowel Disease: A randomized-controlled trial. Journal of Veterinary Internal Medicine 2010;24:269-277.

13. Parente F, Greco S, Molteni M, Aderloni A, Maconi G, Porro GB. Modern imaging off Crohn's disease using bowel ultrasound. Inflammatory Bowel Disease 2004; 10:452-461.

14. Larson MM, Biller DSUltrasound of the gastrointestinal tract. Veterinary Clinics of North America: Small Animal Practice 2009;39:747-759.

15. Agthe P. Ultrasonography of the gastrointestinal tract and associated organs in dogs and cats. In Practice 2009;31:182-188.

16. D'anjou MA. Abdominal cavity, lymph nodes, and great vessels. In: Penninck, D.G. and M.A. D'anjou, (eds). Atlas of small animal ultrasonography. $1^{\text {st }}$ edition. Blackwell Publishing, Iowa 2008, 445-63. 